\title{
Homocysteine in dogs with systemic inflammatory response syndrome
}

\author{
B. E. Patterson*, J. W. Barr*, G. T. Fosgate $\dagger$, N. Berghoff*, J. M. Steiner*, J. S. \\ Suchodolski*, D. M. Black* \\ *Texas A\&M College of Veterinary Medicine, College Station, TX 77843, USA \\ $\dagger$ Production Animal Studies, Faculty of Veterinary Science, University of Pretoria, Onderstepoort, South \\ Africa
}

\begin{abstract}
Objectives - To compare serum concentrations of homocysteine (Hcy) in dogs fitting the criteria for the systemic inflammatory response syndrome (SIRS) and healthy dogs, and compare these values to commonly measured B-vitamins.

Methods - Study dogs were classified into noninfectious SIRS or sepsis groups and blood was drawn on Day 1 of the patient's hospitalization for the measurement of Hcy, folate and cobalamin concentrations. Hcy was measured in 51 clinically normal dogs to serve as the control group.

Results - A statistically significant difference was found between the Hcy concentrations of the healthy group when compared to noninfectious SIRS and sepsis groups. Hcy values were not correlated with folate, cobalamin or APPLE $E_{\text {fast }}$ severity scores. Hcy concentrations were significantly lower in sick dogs when compared to the control group, which is dissimilar to the human population.

Clinical Significance - The clinical significance of Hcy changes in critically ill dogs is currently unknown.
\end{abstract}

Keywords: Homocysteine, B-vitamins, systemic inflammatory response syndrome, sepsis, dogs 


\section{Introduction}

Homocysteine (Hcy), a toxic, sulfur containing amino acid, is an intermediary product of the conversion of the essential amino acid methionine to the antioxidant glutathione. Hcy is tightly regulated through two metabolic pathways, remethylation and transsulfuration (Schindler et al. 2000), and both pathways rely on adequate stores of three dietary vitamins: folate (vitamin $\mathrm{B}_{9}$ ), cobalamin (vitamin $\mathrm{B}_{12}$ ), and pyridoxine (vitamin $\mathrm{B}_{6}$ ) (Fig 1). Defects in these pathways, as well as vitamin deficiencies, age, gender, and renal dysfunction have been linked to elevations in Hcy concentrations and represent an independent risk factor for vascular disease in humans (Refsum et al. 1998).

Hyperhomocysteinemia in people (Hcy $>15 \mu \mathrm{mol} / \mathrm{L})$ is well known to be associated with an increased risk for cardiovascular disease, stroke, peripheral arterial occlusive disease and venous thrombosis (Mizock 2000, Stanger et al. 2003) and may reflect inadequate dietary supply of vitamin $\mathrm{B}_{6}$, folate and cobalamin (McCully 2007). The procoagulant effects of Hcy are ill defined but have been linked to inhibition of thrombomodulin and protein C activation (Hayashi et al. 1992), impairment of endothelial nitric-oxide dependent vasodilation (Jakubowski 2004), and stimulation of tissue factor production (Fryer et al. 1993, Eldibany and Caprini 2007).

Homocysteine has been further investigated for its role in inflammation and atherosclerosis, and appears to be associated with the mechanisms of cellular apoptosis and oxidant stress (Maron and Loscalzo 2009). Homocysteine has been linked to proinflammatory states through upregulation of NF- $\kappa$ B and release of IL-8 (Poddar et al. 2001) and direct damage to endothelial cells (Starkenbaum and Harlan 1986, Chambers 


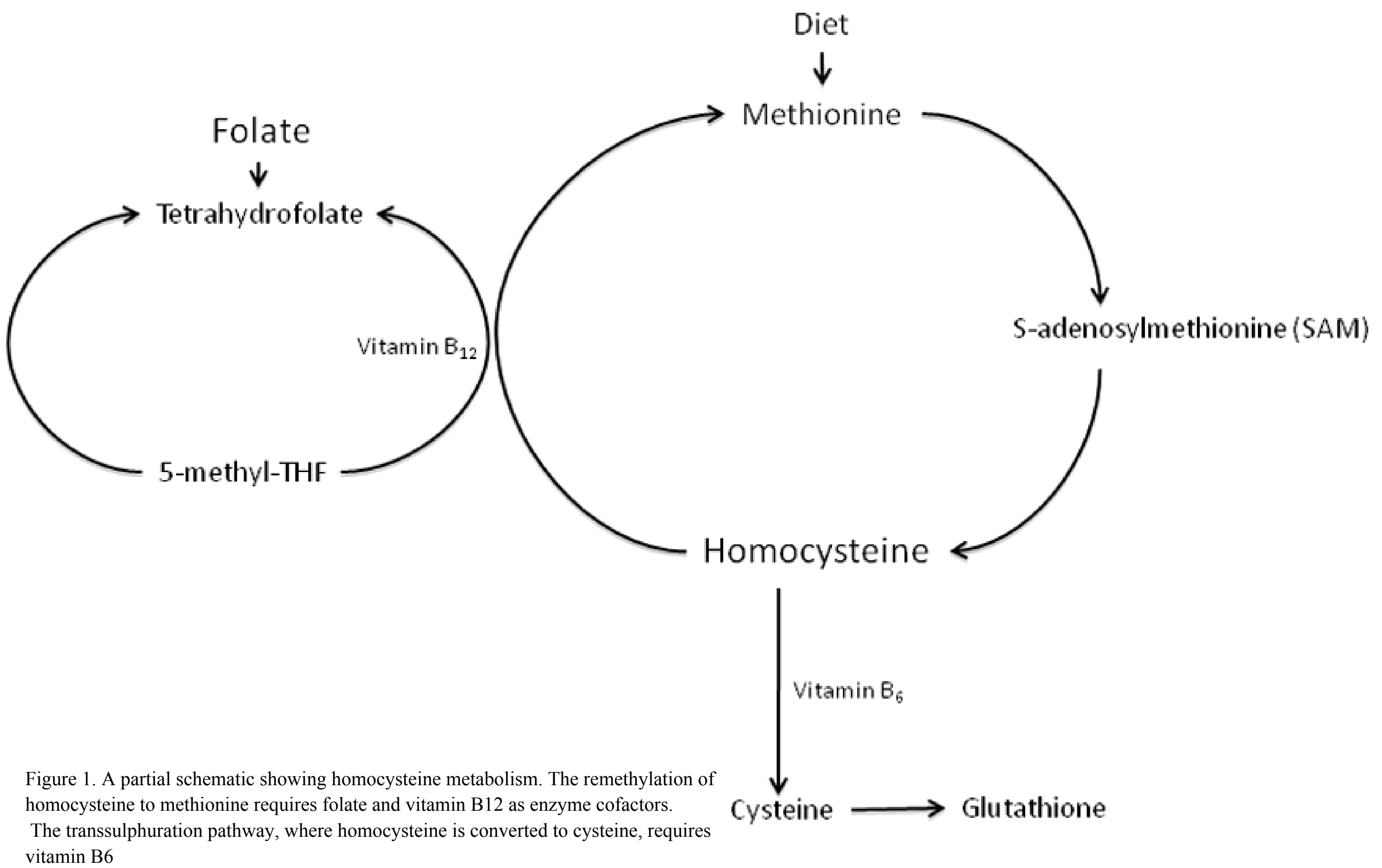


et al. 1999, Austin et al. 2004). Further, Hcy metabolism seems to be influenced by modulation of the immune system (Schroecksnadel 2003).

As the vascular endothelium is often compromised in systemic inflammation (Pinksy and Matuschak 1989, McGill et al. 1998), efforts have been made to assess Hcy concentrations in patients with critical illness. Hyperhomocysteinemia is more commonly observed in critically ill people when compared to age and gender matched healthy individuals (Schindler et al. 2000). Ploder et al (2010) further defined the importance of hyperhomocysteinemia in critically ill humans by documenting changes of Hcy over time of hospitalization, and found significantly higher Hcy concentrations in nonsurvivors as compared to survivors in septic patients.

Although Hcy toxicity is multifactorial and incompletely understood, it appears that Hcy concentration may serve as a marker of endothelial dysfunction and may be associated with outcome. The purpose of this study was to compare Hcy concentrations of dogs suffering from the systemic inflammatory response syndrome (SIRS) to that of clinically healthy dogs. Additionally, we sought to document a correlation of serum Hcy and folate/cobalamin concentrations in sick dogs. We hypothesized that critically ill patients would have an increased serum Hcy concentrations as compared to clinically normal dogs, and that dogs with an increased Hcy concentration would have decreased serum concentrations of cobalamin and folate. Finally, we investigated the human cut-off value of hyperhomocysteinemia (Hcy > $15 \mu \mathrm{mol} / \mathrm{L}$ ) in sick and clinical healthy dogs, to assess its validity in veterinary patients. 


\section{Material \& Methods}

Dogs suffering from the systemic inflammatory response syndrome that were admitted to the intensive care unit from May 2010 to December 2010 were eligible for the study. Inclusion criteria were dogs over $10 \mathrm{~kg}$, to best ensure that no complications would arise from hypovolemia associated with the volume of blood removed, and demonstrating 2 or more of the following SIRS criteria: hypothermia $\left(<37.8^{\circ} \mathrm{C}\right)$, hyperthermia $\left(>39.4^{\circ} \mathrm{C}\right)$, tachycardia $(>140 \mathrm{bpm})$, tachypnea $(>20 \mathrm{bpm})$, leukopenia $\left(<6.0 \times 10^{9} / \mathrm{L} \mathrm{WBC}\right)$, leukocytosis $\left(>16.0 \times 10^{9} / \mathrm{L} \mathrm{WBC}\right)$, or $>3 \%$ immature neutrophils (Hauptman et al. 1997, de Laforcade et al. 2003). Dogs were grouped into noninfectious SIRS or sepsis categories based on the absence or presence of infection (bacterial or viral), respectively. Infection was determined by identification of bacteria via cytology, culture, serology, or with a positive viral antigen test (IDEXX). The primary clinician directed the treatment regimen and there were no interventions as part of the study. Disease severity was assessed within the first 24 hours of hospitalization

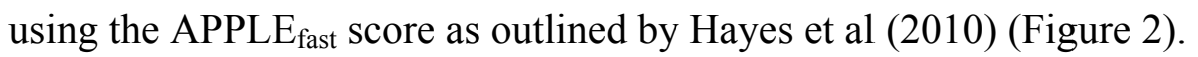

\begin{tabular}{|c|c|c|c|c|c|c|c|c|}
\hline $\begin{array}{l}7 \\
<4.67\end{array}$ & \begin{tabular}{|l|}
8 \\
$4.67-5.67$
\end{tabular} & $\begin{array}{l}9 \\
5.72-9.11\end{array}$ & $\begin{array}{l}10 \\
9.17-15.17\end{array}$ & $\begin{array}{l}\text { glucose }(\mathrm{mmol} / \mathrm{L}) \\
>15.17\end{array}$ & & & & \\
\hline \multirow[t]{4}{*}{$\begin{array}{l}8 \\
<26\end{array}$} & $\begin{array}{l}7 \\
26-30\end{array}$ & \begin{tabular}{|l|l}
6 \\
$31-32$
\end{tabular} & & $\begin{array}{l}\text { albumin }(\mathbf{g} / \mathbf{L}) \\
33-35\end{array}$ & $\begin{array}{l}2 \\
>35\end{array}$ & & & \\
\hline & & & & $\begin{array}{l}\text { lactate }(\mathrm{mmol} / \mathrm{L}) \\
<2\end{array}$ & $\begin{array}{l}4 \\
2-8\end{array}$ & $\begin{array}{l}8 \\
8-10\end{array}$ & $\begin{array}{l}12 \\
>10\end{array}$ & \\
\hline & $\begin{array}{l}\mathbf{5} \\
<151\end{array}$ & $\begin{array}{l}6 \\
151-200\end{array}$ & $\begin{array}{l}3 \\
201-260\end{array}$ & $\begin{array}{l}\text { platelet count }\left(\times 10^{9} / \mathrm{L}\right) \\
261-420,000\end{array}$ & $\begin{array}{l}1 \\
>420\end{array}$ & & & \\
\hline & & & & $\begin{array}{l}\text { mentation score } \\
0\end{array}$ & $\begin{array}{l}4 \\
1\end{array}$ & $\begin{array}{l}6 \\
2\end{array}$ & $\begin{array}{l}7 \\
3\end{array}$ & $\begin{array}{l}14 \\
4\end{array}$ \\
\hline
\end{tabular}

Figure 2. Canine acute patient physiologic and laboratory evaluation (APPLEFAST) severity illness score, as adapted from Hayes et al. (2010). The score is used to stratify illness using 5 patient variables: glucose, albumin, mentation score, platelet count and lactate. The numbers in the upper left corner of each cell show the appropriate score for the relevant variable range. The final score for the patient is obtained by summing the scores for each variable, with a maximum score of 50 
Fifty-one healthy dogs belonging to staff and students of the teaching hospital served as the control group of this study with the goal of having twice as many controls as the number of sick dogs. These dogs were deemed healthy based on physical examination, hematology, and blood chemistry evaluation. Blood was obtained from a single jugular venipuncture and no restrictions were made for fasting of the animals or time of sample collection. No interventions were performed on the healthy dogs.

The study protocol was approved by the hospital's Clinical Research Review Committee (protocol \#10-18) and written owner consent was obtained before subject enrollment. The same sample collection procedures were employed for noninfectious SIRS and sepsis groups. Study dogs had blood drawn on day 1 of hospitalization and this blood draw coincided with other clinically necessary phlebotomy when at all possible. Two milliliters of whole blood was collected into sterile tubes without anticoagulant either from a central intravenous catheter in the hospitalized patients, or via venipuncture in the normal patients. After a firm clot had formed, the tubes were then centrifuged at 1,300 RCF for 10 minutes to separate the serum from the red cell mass. Serum was decanted and stored at $-80^{\circ} \mathrm{C}$ until testing.

\section{$\underline{\text { Sample Analysis }}$}

Serum Hcy concentrations were measured using a stable isotope dilution gas chromatography/mass spectrometry (GC/MS) method, as previously described (Stabler et al. 1987, Ruaux et al. 2001). Assay performance was verified by use of standards prepared with pure homocystine (Sigma-Aldrich) in serial dilutions ranging from 100 $\mu \mathrm{mol} / \mathrm{L}$ to $1.56 \mu \mathrm{mol} / \mathrm{L}$, which correspond to Hcy concentrations of $200 \mu \mathrm{mol} / \mathrm{L}$ to 3.12 
$\mu \mathrm{mol} / \mathrm{L}$. The remaining serum from day 1 was used to measure folate and cobalamin concentrations using a competitive binding chemiluminescence assay (Siemens Medical Solutions Diagnostics). The lower and upper detection limits of the folate assay are 1 and $24 \mu \mathrm{g} / \mathrm{L}$, respectively. The laboratory's reference interval for serum folate concentrations in dogs is $7.7-24 \mu \mathrm{g} / \mathrm{L}$. The lower and upper detection limits for the cobalamin assay are $150-1,000 \mathrm{ng} / \mathrm{L}$; for sample results lower than the detectable range, $150 \mathrm{ng} / \mathrm{L}$ was used for statistical analysis. The laboratory reference range for serum cobalamin concentrations in dogs is $251-908 \mathrm{ng} / \mathrm{L}$.

\section{$\underline{\text { Statistical Analysis }}$}

Data were assessed for normality using descriptive statistics, histograms, and the Anderson-Darling test for normality. Data were presented as median and interquartile range for data violating the normality assumption. Categorical data were compared among noninfectious SIRS, sepsis, and normal dog groups using chi-square tests. Continuous data were compared among groups using Kruskal-Wallis tests with post-hoc Mann-Whitney U tests employing Bonferroni correction for multiple comparisons. The cut off value of hyperhomocysteinemia in humans $(\mathrm{Hcy}>15 \mu \mathrm{mol} / \mathrm{L})$ was evaluated for frequency within our study population and control group. Correlations between variables were estimated using Spearman's rho. Data were analyzed in commercially available software (IBM SPSS) and alpha level of significance assessed at $\mathrm{P}<0.05$. 


\section{Results}

Eleven dogs with noninfectious SIRS, eleven dogs with sepsis, and fifty-one control dogs were enrolled in the study. The noninfectious SIRS group consisted of 6 spayed females, 1 intact female, 2 neutered males, and 2 intact males with ages ranging from 5 months to 11 years. Breeds included 2 German Shepherd dogs and one of each of the following: Australian Kelpie, Bloodhound, Boxer, Brittany Spaniel, English Bulldog, Great Dane, Labrador Retriever, Rottweiler, and Weimaraner. Noninfectious SIRS was assessed to be the result of gastrointestinal obstruction $(n=3)$, gastrointestinal ulceration $(n=1)$, pancreatitis $(n=1)$, pleuritis $(n=1)$, soft tissue trauma $(n=1)$, pancreatic adenocarcinoma $(n=1)$, lymphoma $(n=1)$, immune-mediated hemolytic anemia $(n=1)$ or fever of unknown origin $(n=1)$. The sepsis group consisted of 4 spayed females, 5 neutered males, and 2 intact males. Breeds included 2 mixed breed dogs and one of each of the following: Border Collie, Boxer, Collie, Dogo Argentino, German Shepherd dog, Labrador Retriever, Rottweiler, and Sheltie. Sepsis was caused by septic peritonitis $(n=5)$, parvovirus enteritis $(n=3)$, pyelonephritis $(n=1)$, body wall abscess $(n=1)$ and Rocky Mountain Spotted Fever $(n=1)$. The healthy control group consisted of 23 spayed females, 1 intact female, 25 neutered males, and 2 intact males of various breeds, ranging from 6 months to 13 years of age. Sex $(P=0.567)$ and age $(P=0.635)$ did not vary between healthy and sick patients. Sickness severity, as assessed by the APPLE $_{\text {fast }}$ scores, did not differ statistically between the noninfectious SIRS and sepsis groups $(P=0.438)$ (Table 1$)$.

Median Hcy concentrations of healthy dogs $(11.1 \mu \mathrm{mol} / \mathrm{L}$; IQR 8.89, 17.20) were significantly higher than noninfectious SIRS (7.3 $\mu \mathrm{mol} / \mathrm{L}$; IQR 5.17, 10.90) 
Table 1. Homocysteine, APPLE fast scores, folate and cobalamin concentrations for SIRS, sepsis, and control groups.

\begin{tabular}{|c|c|c|c|c|c|c|}
\hline \multirow[b]{2}{*}{ Variable } & \multicolumn{2}{|c|}{ SIRS (n=11) } & \multicolumn{2}{|c|}{ Sepsis $(n=11)$} & \multicolumn{2}{|c|}{ Normal $(n=51)$} \\
\hline & $\overline{\text { Median (n) }}$ & IQR & $\overline{\text { Median (n) }}$ & IQR & $\overline{\text { Median (n) }}$ & IQR \\
\hline $\begin{array}{l}\text { Homocysteine }(\mu \mathrm{mol} / \mathrm{L}) \\
(\mathrm{p}<0.05)\end{array}$ & $7.26(11)$ & $5.17,10.9$ & $4.70(10)$ & $3.41,9.66$ & $11.1(51)$ & $8.89,17.2$ \\
\hline $\begin{array}{l}\text { APPLE }{ }_{\text {fast }} \text { Scores } \\
(\mathrm{P}=0.438)\end{array}$ & $24(11)$ & $21.0,29.5$ & $26(11)$ & $20.0,26.5$ & $\mathrm{n} / \mathrm{a}$ & $\mathrm{n} / \mathrm{a}$ \\
\hline $\begin{array}{l}\text { Folate }(\mu \mathrm{g} / \mathrm{L}) \\
(\mathrm{P}=0.77)\end{array}$ & $6.77(10)$ & $4.25,7.75$ & $5.32(11)$ & $3.69,8.29$ & $\mathrm{n} / \mathrm{a}$ & $\mathrm{n} / \mathrm{a}$ \\
\hline $\begin{array}{l}\text { Cobalamin }(\mathrm{ng} / \mathrm{dL}) \\
(\mathrm{P}=0.435) \\
\text { IOR = interquartile range }\end{array}$ & $242.5(10)$ & 216,331 & $205(11)$ & 183,327 & $\mathrm{n} / \mathrm{a}$ & $\mathrm{n} / \mathrm{a}$ \\
\hline
\end{tabular}




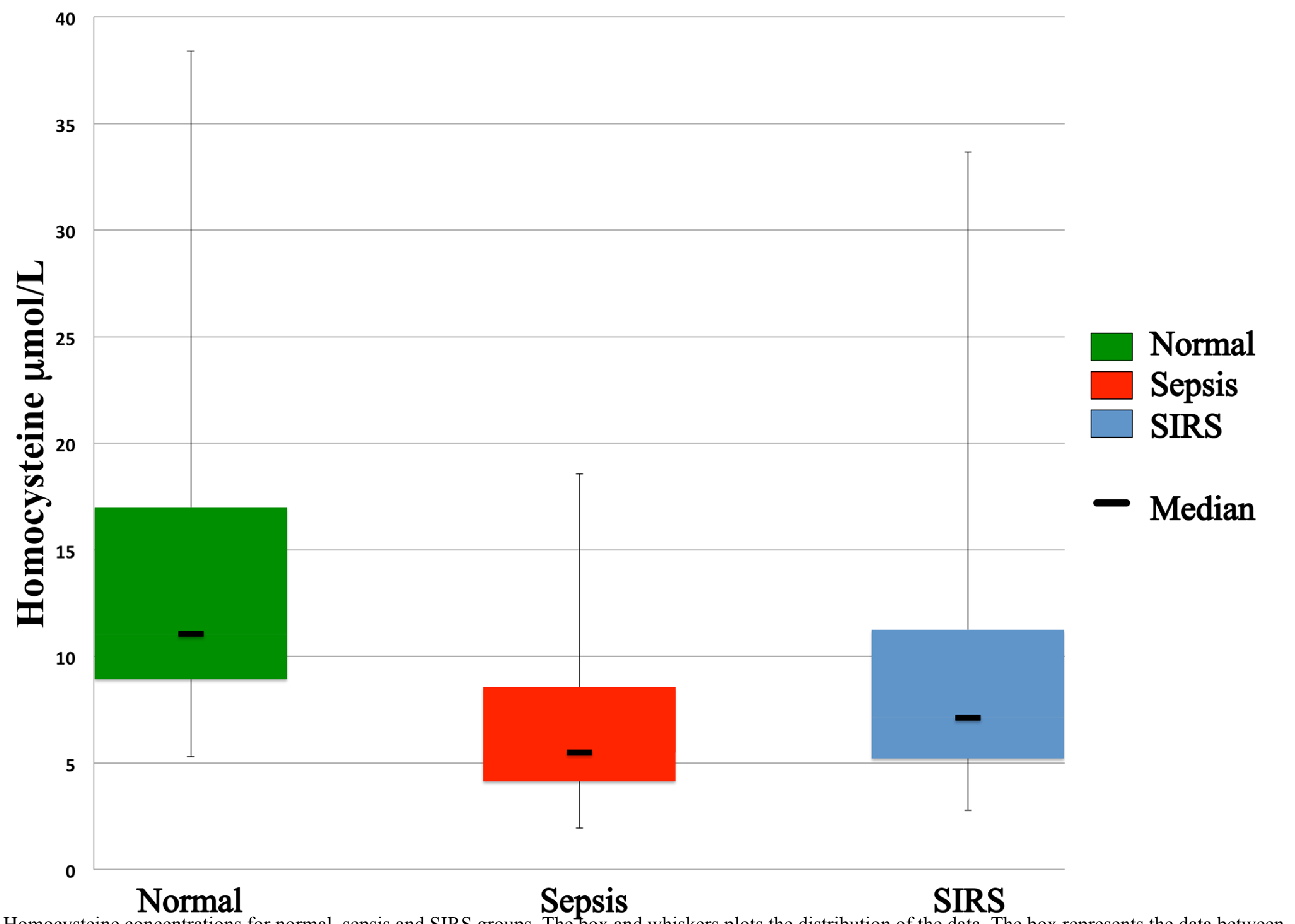

Figure 3. Homocysteine concentrations for normal, sepsis and SIRS groups. The box and whiskers plots the distribution of the data. The box represents the data between the upper and lower quartile while the whiskers extend to the minimum and maximum of the data 
and sepsis $(4 \cdot 7 \mu \mathrm{mol} / \mathrm{L}$; IQR $3 \cdot 41,9 \cdot 66)$ groups_from Day 1 (Table 1, Figure 3).

APPLE $_{\text {fast }}$ severity scores were not correlated with Hcy levels within the noninfectious SIRS and sepsis groups $(\rho=0.393 ; P=0.078)$. Hcy concentrations did not correlate with folate $(\rho=-0.057 ; P=0.811)$ nor cobalamin $(\rho=0.146 ; P=0.538)$ concentrations. Decreased folate, cobalamin or both were present in $95 \%(20 / 21)$ of the sick patients. The vitamins were evaluated in only 10 of the 11 noninfectious SIRS dogs, as one sample could not be run due to an insufficient quantity of serum. Human defined hyperhomocysteinemia was more prevalent within the healthy dogs as compared to the sick dogs $(P=0.005)$.

\section{Discussion}

In our study, Hcy concentrations of dogs hospitalized for noninfectious SIRS and sepsis were significantly different from clinically healthy dogs. However, unlike human critically ill patients (Schindler et al. 2000), sick dogs in our study had lower Hcy concentrations compared to healthy dogs. The cause of this variation remains to be determined and may be multifactorial. Dogs may possess different mechanisms for Hcy concentration regulation during periods of inflammation and infection. Alternatively, in states of nutritional stress, humans and dogs may conserve amino acid profiles differently. For example, Chan et al (2009) found that the amino acid methionine was significantly lower in critically ill dogs. It is possible that minimal dietary intake was the basis of this finding, however, nutritional status was not assessed. Multiple human studies have documented the strong correlation with increased Hcy levels following methionine loading (de Bree et al. 2002, Stanger et al. 2003). Therefore, it is prudent to 
recognize the possibility of low methionine concentrations, possibly from low dietary intake, playing a role in the low Hcy values seen within our sick patients. Variances in human versus veterinary therapy may also explain differences in amino acid metabolism and profiles in disease.

We found no correlation between Hcy concentrations and cobalamin/folate concentrations, even though significant vitamin deficiencies were evident in the noninfectious SIRS and septic dogs. This too is inconsistent with human data, as cobalamin and folate deficiencies are well known acquired and genetic risk factors for hyperhomocysteinemia in humans (Clarke et al. 1991, Graham et al. 1997, Armitage et al. 2010). It is possible that cobalamin and folate deficiencies of critical illness are too acute to lead to detectable hyperhomocysteinemia within the first few days of disease. This could also represent a distinct metabolic difference between humans and dogs. This warrants further investigation.

This study found that Hcy concentrations $>15 \mu \mathrm{mol} / \mathrm{L}$ were more likely to occur in healthy rather than critically ill dogs, which is in direct opposition to human literature. Only one noninfectious SIRS patient ( $4.5 \%$ of sick dogs) had a Hcy concentration greater than $15 \mu \mathrm{mol} / \mathrm{L}$, whereas $37 \%$ (19/51) of healthy dogs had a Hcy concentration greater than $15 \mu \mathrm{mol} / \mathrm{L}$. This may indicate that this cut-off lacks clinical relevance, based on the canine patients in our study. In a recent publication, Ploder et al (2010) documented a direct correlation between the degree of hyperhomocysteinemia (Hcy $>15 \mu \mathrm{mol} / \mathrm{L}$ ) and disease severity in critically ill humans. In fact, the patients in the study by Ploder et al with the highest Hcy concentrations showed the greatest mortality rates (2010). Understanding of this distinct difference between critically ill 
dogs and humans could prove worthwhile for people at high risk of cardiovascular disease and peripheral arterial occlusion. It could be that humans tend to suffer from more chronic diseases than our canine patients and Hcy levels reflect the comorbidity of endothelial damage over time, leading to changes of Hcy in humans that would not replicated in dogs. There has also been a wide variation in Hcy concentrations reported in normal dogs (Rossi et al. 2008). Trisolini et al (2008) documented that intact females in anestrus and spayed females have significantly higher Hcy concentrations compared to cycling or pregnant bitches. Although stage of estrus is known to affect Hcy concentrations, as our study population contained only two intact females, one in the sick population and one in the healthy population, and no statistically significant difference was present Hcy between sexes, the role of estrus on the Hcy concentration was not investigated further in this study. The Hcy values greater than $15 \mu \mathrm{mol} / \mathrm{L}$ in these healthy dogs may reflect a normal post-prandial response, as all dogs were not fasted prior to blood draw. In order to resolve this variation, a controlled study to document Hcy concentrations in fasted as well as post-prandial settings is warranted.

Limitations of our study included the small number of affected dogs in the noninfectious SIRS and sepsis groups, potentially leaving the data underpowered to detect differences between groups. Also, although we believe that our grouping of noninfectious SIRS and sepsis was correct, the possibility exists of not defining an infection when one was indeed present. Another inherent difficulty when assessing data in sick patients is recognizing that disease severity between patients may dictate the final results. We attempted to minimize this potential limitation by using the previously 
validated APPLE $_{\text {fast }}$ score (Hayes 2010) to account for the patient's level of sickness and improve comparative validity.

In conclusion, our study found that serum Hcy concentrations in dogs with noninfectious SIRS or sepsis were significantly lower than those in healthy dogs, and Hcy did not correlate with folate/cobalamin concentrations. The clinical significance of this is unknown, however, a more complete understanding of the differences between critically ill dogs and humans may provide insight into treatment of hyperhomocysteinemia in humans.

\section{$\underline{\text { References }}$}

1. Armitage, J.M., Bowman, L., Clarke, R.J. (2010) Effects of homocysteinelowering with folic acid plus vitamin $B_{12}$ vs placebo on mortality and major morbidity in myocardial infarction survivors. Journal of the American Medical Association 303, 2486-2494

2. Austin, R.C., Lentz, S.R., Werstuck, G.H. (2004) Role of hyperhomocysteinemia in endothelial dysfunction and atherothrombotic disease. Cell Death and Differentiation 11, S56-S64

3. Chambers, J.C., McGregor, A., Jean-Marie, J., et al. (1999) Demonstration of rapid onset vascular endothelial dysfunction after hyperhomocysteinemia: an effect reversible with vitamin C therapy. Circulation 99, 1156-1160

4. Chan, D.L., Rozanski, E.A., Freeman, L.M. (2009) Relationship among plasma amino acids, C-reactive protein illness severity, and outcome in critically ill dogs. Journal of Veterinary Internal Medicine 23, 559-563 
5. Clarke, R., Daly, L., Robinson, K., et al. (1991) Hyperhomocysteinemia: an independent risk factor for vascular disease. New England Journal of Medicine 324, $1149-1155$

6. de Bree, A., Verschuren, W.M.M., Kromhout, D., et al. (2002) Homocysteine determinants and the evidence to what extent homocysteine determines the risk of coronary heart disease. Pharmacological Reviews 54, 599-618

7. de Laforcade, A.M., Freeman, L.M., Shaw, S.P., et al. (2003) Hemostatic changes in dogs with naturally occurring sepsis. Journal of Veterinary Internal Medicine 17, 674-679

8. Eldibany, M.M., Caprini, J.A. (2007) Hyperhomocysteinemia and thrombosis. Archives of Pathology and Laboratory Medicine 131, 872-884

9. Fryer, R.H., Wilson, B.D., Gubler, D.B., et al. (1993) Homocysteine, a risk factor for premature vascular disease and thrombosis, induces tissue factor activity in endothelial cells. Arteriosclerosis, Thrombosis, and Vascular Biology 13, $1327-$ 1333

10. Graham, I.M., Daly, L.E., Refsum, H.M., et al. (1997) Plasma homocysteine as a risk factor for vascular disease. The European Concerted Action Project. Journal of the American Medical Association 277, 1775-1781

11. Hauptman, J.G., Walshaw, R., Olivier, N.B. (1997) Evaluation of the sensitivity and specificity of diagnostic criteria for sepsis in dogs. Veterinary Surgery 26, 393-397

12. Hayashi, T., Honda, G., Suzuki, K. (1992) An atherogenic stimulus homocysteine inhibits cofactor activity of thrombomodulin and enhances thrombomodulin expression in human umbilical vein endothelial cells. Blood 79, 2930-2936 
13. Hayes, G., Mathews, K., Doig, D., et al. (2010) The acute patient physiologic and laboratory evaluation (APPLE) score: a severity of illness stratification system for hospitalized dogs. Journal of Veterinary Internal Medicine 24, 1034-1047

14. Jakubowski, H. (2004) Molecular basis of homocysteine toxicity in humans. Cellular and Molecular Life Sciences 61, 470-487

15. Maron, B.A., Loscalzo, J. (2009) The treatment of hyperhomocysteinemia. Annual Review of Medicine 60, 39-54

16. McCully, K.S. (2007) Homocysteine, vitamins, and vascular disease prevention. American Journal of Clinical Nutrition 86, 1563S-1568S

17. McGill, S.N., Ahmed, N.A., Christou, N.V. (1998) Endothelial cells: role in infection and inflammation. World Journal of Surgery 22, 171-178

18. Mizock, B.A. (2000) Homocysteine and critical illness. Critical Care Medicine 28, $1229-1230$

19. Ploder, M., Kurz, K., Spittler, A., et al. (2010) Early increase of plasma homocysteine in sepsis patients with poor outcome. Molecular Medicine 16, 498504

20. Poddar, R., Sivasubramanian, N., DiBello, P.M., et al. (2001) Homocysteine induces expression and secretion of monocyte chemoattractant protein- 1 and interleukin-8 in human aortic endothelial cells: implications for vascular disease. Circulation 103, 2717-2723

21. Ruaux, C.G., Steiner, J.M., Williams, D.A. (2001) Metabolism of amino acids in cats with severe cobalamin deficiency. American Journal of Veterinary Research $62,1852-1858$ 
22. Refsum, H., Ueland, P.M., Nygard, O., et al. (1998) Homocysteine and cardiovascular disease. Annual Review of Medicine 49, 31-62

23. Rossi, S., Rossi, G., Giordano, A., et al. (2008) Homocysteine measurement by an enzymatic method and potential role of homocysteine as a biomarker in dogs. Journal of Veterinary Diagnostic Investigation 20, 644-649

24. Schindler, K., Zauner, C., Buchmayer, H., et al. (2000) High prevalence of hyperhomocysteinemia in critically ill patients. Critical Care Medicine 28, 991-995

25. Schroecksnadel, K., Frick, B., Wirleitner, B., et al. (2003) Homocysteine accumulates in supernatants of stimulated human peripheral blood mononuclear cells. Clinical and Experimental Immunology 134, 53-56

26. Stabler, S.P., Marcell, P.D., Podell, E.R., et al. (1987) Quantitation of total homocysteine, total cysteine, and methionine in normal serum and urine using capillary gas chromatography-mass spectrometry. Anaytical Biochemistry 162, 185196

27. Stanger, O., Herrmann, W., Pietrzik, K., et al. (2003) DACH-LIGA Homocystein (German, Austrian and Swiss Homocysteine Society): Consensus paper on the rational clinical use of homocysteine, folic acid and B-vitamins in cardiovascular and thrombotic diseases: guidelines and recommendations. Clinical Chemistry and Laboratory Medicine 41, 1392-1403

28. Starkenbaum, G., Harlan, J.M. (1986) Endothelial cell injury due to coppercatalyzed hydrogen peroxide generation from homocysteine. Journal of Clinical Investigation 77, 1370-1376 
29. Trisolini, C., Minoia, G., Manca, R. et al. (2008) Plasma homocysteine levels in cycling, pregnant, and spayed bitches. Animal Reproduction Science 108, 29-36 